\author{
ANNALES \\ POLONICI MATHEMATICI \\ XXXVIII (1980)
}

\title{
A new characterization of the sphere in $R^{3}$
}

\author{
by Thomas Hasanis (Ioannina, Greece)
}

\begin{abstract}
Let $M$ be a closed connected surface in $R^{3}$ with positive Gaussian curvature $K$ and let $K_{I I}$ be the curvature of its second fundamental form. It is shown that $M$ is a sphere if $K_{I I}=c \sqrt{H} K^{r}$, for some constants $c$ and $r$, where $H$ is the mean curvature of $\boldsymbol{M}$.
\end{abstract}

1. Introduction. A closed connected surface in $R^{3}$ with Gaussian curvature $K>0$ throughout, an ovaloid in short, possesses a positivedefinite second fundamental form $I I$, if appropriately oriented. We denote by $K_{I I}$ the Gaussian curvature of the second fundamental form $I I$ and by $H$ the mean curvature of $M$. Many authors have concerned themselves with the problem of characterization of the sphere by the curvature of the second fundamental form. In [2] Schneider has shown that the constancy of the curvature $K_{I I}$ implies that $M$ is a sphere. Koutroufiotis [1] has shown that $M$ is a sphere if $K_{I I}=c K$ or $K_{I I}=\sqrt{K}$ for some constant $c$. Stamou [3] has shown that $M$ is a sphere if $K_{I I}=c H \sqrt{K}$ or $K_{I I}=c H / \sqrt{\bar{K}}$ for some constant $c$. Also Stamou shows in [3] that $M$ is a sphere if $K_{I I}$ $=c K^{r}$, for some constants $c$ and $r$. This last result gives the results of [1] and [2] for appropriate constants $c$ and $r$.

The purpose of this note is to prove the following

MaIN Theorem. Let $M$ be an ovaloid in $R^{3}$. If $K_{I I}=c \sqrt{H} K^{r}$, where 0 and $r$ are constants, then $M$ is a sphere.

Remark. Obviously the constant $c$ must be positive, as follows from the Gauss-Bonnet theorem.

2. Preliminaries. Let $\left(u^{i}\right)$ be local coordinates on $M$ and let $\Gamma_{i j}^{k}, \nabla_{I}$ [resp. ${ }_{I I} \Gamma_{i j}^{k}, \nabla_{I I}$ ] denote the Christoffel symbols and the first Beltrami operator relative to the first fundamental form $I$ [resp. to the second fundamental form $I I]$. If

$$
T_{i j}^{k}=\Gamma_{i j}^{k}-{ }_{11} \Gamma_{i j}^{k} \quad(i, j, k=1,2),
$$

then by making use of the second fundamental tensor $b_{i j}$ for "raising and lowering indices" we conclude ([2]) that $T_{i j k}=T_{i j}^{h} b_{h k}$ is totally symme- 
tric and

$$
K_{I I}=H+\frac{1}{2} T_{i j k} T^{i j k}-\left(\frac{1}{8 K^{2}}\right) \nabla_{I I} K
$$

3. Main results. If we denote by $d A$ and $d A_{I I}$ the area elements of $M$ with respect to the first and the second fundamental form, it is obvious that $d A_{I I}=\sqrt{K} d A$. Then by the Gauss-Bonnet theorem we have

$$
4 \pi=\int_{M} K d A=\int_{M} K_{I I} d A_{I I}=\int_{M} K_{I I} \sqrt{\bar{K}} d A
$$

or

$$
\int_{\mathbf{I}} \sqrt{K}\left(\sqrt{K}-K_{I I}\right) d A=0
$$

Proof of the Main Theorem. From equation (2) for $K_{I I}=c \sqrt{H} K^{r}$ we get

$$
\int_{M} \sqrt{K}\left(K-c \sqrt{\bar{H}} K^{r}\right) d A=0
$$

Now, let $P_{1}$ be a critical point of $K$; then by (1) we have

$$
K_{I I}\left(P_{1}\right) \geqslant H\left(P_{1}\right)
$$

or

$$
c \sqrt{H\left(P_{1}\right)} K^{r}\left(p_{1}\right) \geqslant H\left(P_{1}\right) \geqslant \sqrt{K\left(P_{1}\right)}, \quad \text { since } H \geqslant \sqrt{\bar{K}} \text {. }
$$

Hence

$$
\sqrt{H\left(P_{1}\right)}\left(c K^{r}\left(P_{1}\right)-\sqrt{H\left(P_{1}\right)}\right) \geqslant 0
$$

and so

$$
\text { cK }^{r}\left(P_{1}\right) \geqslant \sqrt{H\left(P_{1}\right)}, \quad \text { since } \sqrt{H\left(P_{1}\right)}>0 .
$$

Moreover, since $\sqrt{H} \geqslant K^{1 / 4}$ everywhere on $M$, we obtain the following relation :

$$
c K^{r}\left(P_{1}\right) \geqslant \sqrt{H\left(P_{1}\right)} \geqslant K^{1 / 4}\left(P_{1}\right)
$$

or

$$
K^{r}\left(P_{1}\right)\left(c-K^{1 / 4}\left(P_{1}\right)\right) \geqslant 0
$$

that is

$$
K^{1 / 4-r}\left(P_{1}\right) \leqslant c,
$$

because $K^{r}\left(P_{1}\right)>0$.

We distinguish the following two cases. 
Case 1 . Let $r \leqslant \frac{1}{4}$ and so $\frac{1}{4}-r \geqslant 0$. In that case, we choose as $P_{1}$ a point such that $K\left(P_{1}\right)=\operatorname{Sup}_{P \in M} K(P)$ (at least one such point $P \in M$ necessarily exists, since $M$ is closed and $K$ is a continuous function). Then from (4) we conclude that

$$
K^{1 / 4-r} \leqslant c \quad \text { everywhere on } M \text {. }
$$

Case 2. Let $r>\frac{1}{4}$ and thus $\frac{1}{4}-r<0$. In that case, we choose as $P_{1}$ a point such that $K\left(P_{1}\right)=\min _{P \in M} K(P)$. Then from (4) we again obtain

$$
K^{1: 1-r} \leqslant c \quad \text { everywhere on } M \text {. }
$$

Generally, we conclude that for all values of $r$ it holds

$$
K^{1 / 4-r} \leqslant c \quad \text { everywhere on } M \text {. }
$$

Moreover, since $\sqrt{H} \geqslant K^{1 / 4}$, we have

that is

$$
K^{1 / 4} \cdot K^{1 / 4-r} \cdot K^{r} \leqslant c \sqrt{H} K^{r}
$$

or, equivalently,

$$
\sqrt{K} \leqslant c \sqrt{H} K^{r}
$$

$$
\sqrt{\bar{K}}-c \sqrt{\bar{H}} K^{r} \leqslant 0 .
$$

Since the function $\sqrt{K}-c \sqrt{H} K^{r}$ is non-positive, we get from (3)

Thus

$$
\sqrt{K}=c \sqrt{H} K^{r} \text {. }
$$

$$
K_{I 1}=c \sqrt{\bar{H}} K^{r}=\sqrt{K} .
$$

But from a well-known result $([1]$, p. 177) we conclude that $M$ is a sphere, because $K_{I I}=\sqrt{K}$.

This completes the proof of the theorem.

\section{References}

[1] D. Koutroufiotis, Two characteristic properties of the sphere, Proc. Amer. Math. Soc. 44 (1974), p. 176-178.

[2] R. Schneider, Olosed convex hypersurfaces with second fundamental form of constant curvature, ibidem 35 (1972), p. 230-233.

[3] G. Stamou, Global characterizations of the sphere (to appear).

DEPARTMENT OF MATHEMATICS, UNIVERSITY OF IOANNINA IOANNINA, GREECE

Reģu par la Rédaction le 2\%. 11. 1976

4 - Annales Polonicl Mathematici XXXVIII.1 